\title{
Intrahepatic IgG/IgM plasma cells ratio helps in classifying autoimmune liver diseases
}

\author{
Daniela Cabibi $^{\mathrm{a}}$, Giuseppe Tarantino ${ }^{\mathrm{b}, *}$, Francesco Barbaria $^{\mathrm{b}}$, Maria Campione ${ }^{\mathrm{a}}$, \\ Antonio Craxì ${ }^{\mathrm{b}}$, Vito Di Marco ${ }^{\mathrm{b}}$ \\ a Dipartimento di Patologia Umana, University of Palermo, Italy \\ b Cattedra e Unità Operativa di Gastroenterologia, University of Palermo, Italy
}

\section{A R T I C L E I N F O}

\section{Article history:}

Received 23 June 2009

Accepted 13 December 2009

Available online 13 January 2010

\section{Keywords:}

Autoimmune cholangitis

Autoimmune hepatitis

IgG plasma cells

IgM plasma cells

Immunostaining

Liver biopsy

Overlap syndromes

Portal infiltrate

Primary biliary cirrhosis

\begin{abstract}
A B S T R A C T
Background/Aim: Plasma cells infiltrate in the liver is a prototype lesion of autoimmune liver diseases. The possible role of plasma cells isotyping (IgM and IgG) in the liver in the diagnostic definition of autoimmune liver disease, and particularly in variant syndromes such as autoimmune cholangitis and the primary biliary cirrhosis/autoimmune hepatitis overlap syndrome, is less defined.

Methods: We analysed the clinical, serological and histological features of 83 patients with autoimmune liver disease ( 40 primary biliary cirrhosis, 20 autoimmune hepatitis, 13 primary sclerosing cholangitis, 4 autoimmune cholangitis and 6 overlap syndrome) compared to 34 patients with chronic hepatitis $C$ and evaluated the expression of IgM and IgG plasma cells in their liver by immunostaining.

Results: By Spearman's correlation, the mean-counts of IgM plasma cells in portal tracts were significantly correlated with female gender, serum alkaline phosphatase, gamma-glutamyl transferase and IgM values, positivity for anti-mitochondrial antibody-M2 and, on liver biopsy, with bile duct changes, orcein-positive granules and granulomas. Whereas IgG plasma cells resulted more correlated with alanine aminotransferase levels. IgG/IgM ratio lower than 1 was found no only in primary biliary cirrhosis but also in all patients with autoimmune cholangitis. Conversely, all patients with overlap syndrome showed IgG/IgM ratio higher than 1.

Conclusion: Immunostaining for IgM and IgG plasma cells on liver tissue can be a valuable parameter for better diagnosis of autoimmune liver disease and also for variant or mixed syndromes.
\end{abstract}

(C) 2009 Editrice Gastroenterologica Italiana S.r.l. Published by Elsevier Ltd. All rights reserved.

\section{Introduction}

Primary biliary cirrhosis (PBC), autoimmune hepatitis (AIH) and primary sclerosing cholangitis (PSC) cover $90 \%$ of the spectrum of autoimmune liver diseases. Diagnosis is based on clinical, biochemical, serological and histological findings [1]. Approximately $10 \%$ of patients with $\mathrm{PBC}$ or AIH are affected by variant syndromes (VS), presenting simultaneous serological and/or histological features of both diseases. The diagnosis of VS can be difficult in clinical practice because there are no standardized definitions. Biochemical and serological criteria are used to characterize the overlap syndrome (OS), and a liver biopsy that reveals biliary duct injuries and moderate to severe interface hepatitis simultaneously [2-4] helps confirming the diagnosis of OS. Likewise, another variant syn-

\footnotetext{
* Corresponding author at: Via Re Federico 13, 90141 Palermo, Italy. Tel.: +39 091655 2280; fax: +390916552223.

E-mail addresses: cabibidaniela@virgilio.it(D. Cabibi),giutar@alice.it (G. Tarantino), fbarbaria@libero.it (F. Barbaria), campione1954@libero.it (M. Campione), craxanto@unipa.it (A. Craxì), vito.dimarco@tin.it (V. Di Marco).
}

drome, called autoimmune cholangitis (AIC) or anti-mitochondrial antibody negative PBC [5], can also be missed on biopsy, particularly when histology lacks florid bile duct lesions and/or other typical lesions that contribute to the histological diagnosis of $\mathrm{PBC}$ $[6,7]$.

Plasma cells infiltrate is a histological feature frequently seen in the liver tissue of patients with autoimmune liver disease. The presence of plasma cells in the portal and peri-portal inflammation infiltrate is a well-known feature of $\mathrm{PBC}$ and of $\mathrm{AIH}$, and a predominance of immunoglobulin $\mathrm{M}$ (IgM) in PBC and of immunoglobin $\mathrm{G}$ (IgG) in AIH has been reported [8,9]. In patients with PBC, owing to an innate immunity-induced hyper-responsive state the serum levels of IgM are elevated [10,11], though the specificity of this test is low; an increase in IgM serum levels is also reported in patients with PSC [12].

Using immuno-histochemical techniques, we analysed the plasma cells infiltrate in liver biopsies of a cohort of patients affected by autoimmune chronic liver disease, evaluating the amount of cells expressing immunoglobulin isotypes M and G (IgM, $\operatorname{IgG})$. The aim of the study was to investigate the relation between plasma cells infiltrate and other clinical and histological features 
of autoimmune liver disease, as well as the possible role of plasma cells infiltrate in the diagnosis of VS.

\section{Patients and methods}

\subsection{Patients}

We prospectively analysed the records of 83 consecutive, untreated patients with clinical, serological and histological features of immune-mediated liver disease referred at our Liver Unit for the liver biopsy between 2004 and 2008, comparing them with 34 consecutive patients with chronic hepatitis $\mathrm{C}(\mathrm{CHC})$ evaluated and underwent liver biopsy between September and December 2008. The diagnosis of $\mathrm{PBC}$ was based on the presence of elevated serum values of alkaline phosphatase (AP) and/or gamma-glutamyl transferase (GGT), anti-mitochondrial antibody (AMA) detectable in the serum, and on liver biopsy showing such suggestive features as bile duct loss, chronic cholangitis, epithelioid granulomas, orcein-positive granules, interface and lobular hepatitis and fibrosis $[1,6,7,13,14]$.

The diagnosis of AIH was made according to the revised criteria of the International Autoimmune Hepatitis Group (IAHG) [15]. Only patients with a score of $>15$, and without previous immunosuppressive therapy, were included in the study. A diagnosis of PSC was made in the presence of characteristic changes in the intrahepatic and extrahepatic biliary tree, seen with direct cholangiographic imaging, and in the presence of compatible histological features [16]. Some patients were considered affected by VS. In particular, a diagnosis of OS was formulated for patients who fulfilled at least two of the three criteria for the diagnosis of PBC, and had clinical and histological features of AIH based on an aggregate revised IAHG score of at least 10 points in the absence of immune-suppressive therapy [17]. A diagnosis of AIC was formulated for patients who showed a predominant biochemical cholestatic expression, and fulfilled at least 2 criteria for diagnosis of PBC, but with an aggregated revised IAHG score $\leq 10$. None of the patients had a previous or current history of alcohol or drug abuse.

HBV chronic hepatitis and genetic liver diseases (Wilson's disease, $\alpha 1$-antitripsin deficit and hereditary haemochromatosis) in the cases were excluded by means of current virological, serological and clinical tests. The study was performed in accordance with the principles of Good Clinical Practice, the principles of the Declaration of Helsinki and its appendices, and local and national laws.

\subsection{Laboratory assessment}

Routine clinical examinations and serological studies were performed for all patients at the time of first admission to our Liver Unit and were available in the hospital files. All patients were tested for antinuclear antibody (ANA), smooth muscle antibody (SMA), liver-kidney microsomal type 1 antibody (LKM1), and AMA, with indirect immunofluorescence method using mouse stomach tissue and kidney tissue section as substrates (Alifax, Padua, Italy). A serum titre of 1:80 or higher was considered positive for ANA and SMA, and a titre of 1:40 or higher was considered positive for anti-LKM1 and AMA. E2 subunit of pyruvate dehydrogenase mitochondrial auto-antigen (M2) was detected by enzyme-linked immunoadsorbent assay (immuno-blotting) against pyruvate dehydrogenase complex (Euroimmun version 2006, Lubeck, Deutschland). Serum IgM levels were measured with standard techniques.

\subsection{Histological evaluation}

Percutaneous liver biopsies, performed with a 16-gauge Menghini needle, were $10 \%$ formalin fixed and paraffin embedded. We used $5 \mu \mathrm{m}$ thick serial sections that were rewashed, rehydrated and stained with haematoxylin-eosin, Shikata's orcein, PAS diastase, and Gomori stain for reticular fibres, and also with Masson's trichromic stain. Slides of liver specimens were coded and re-read by a pathologist (D.C.), who was unaware of patient's identity and clinical or serological features. Only biopsies at least $15 \mathrm{~mm}$ long and containing more than eight portal tracts were evaluated.

As mentioned above we investigated the histological features associated with PBC: chronic non-suppurative destructive cholangitis involving interlobular bile ducts, with typical duct lesions consisting of lymphocytic infiltration, irregularity and eosinophilic changes of the epithelium; and bile duct loss and/or atypical ductular proliferation, which we generally coded as "ductular changes." In addition, we considered other features that helped confirm histological diagnosis, such as portal epithelioid granulomas, which were identified as the presence of small aggregates of histiocytoid cells in the portal tract, often surrounding a biliary duct, and chronic cholestasis, as evidenced by the presence of peri-portal orcein-positive granules [6].

We also investigated the typical histological findings of $\mathrm{AIH}$, such as mononuclear and plasma cells infiltration of the portal spaces expanding into the liver lobule; destruction of the hepatocytes at the periphery of the lobule, with erosion of the limiting plate (interface hepatitis); connective tissue collapse from the portal area into the lobule (bridging collapse); and rosette formation due to hepatic regeneration [15]. Characteristic histological features of PSC were evaluated, such as degeneration and proliferation of epithelial cells of bile ducts, infiltration of the bile ducts by lymphocytes and neutrophils, and the formation of concentric layers of connective tissue surrounding bile ducts (onionskin lesion) [16]. Finally, the grade of interface hepatitis and the stage of fibrosis were evaluated according to the histological scoring system proposed by Hiramatsu et al. [6].

Immunohistochemical staining was performed using the streptavidin-biotin-complex technique. The primary antibodies used in this study were polyclonal antibodies anti-IgG and antiIgM, both diluted 1/2000 (Novocastra, Newcastle, United Kingdom). Before incubation with anti-IgG and anti-IgM antibodies, a pretreatment with pepsin was done to improve the immunostaining. The endogenous peroxidase activity was blocked by incubation of liver sections in a solution of $0.3 \%$ hydrogen peroxidase and absolute methanol. The non-specific antigenic sites were blocked using a protein block solution included in the kit. After incubation for $15 \mathrm{~min}$ at room temperature, the sections were incubated with the secondary antibody, which was followed by streptavidine-biotincomplex. Amino-ethil-carbazole was used as chromogen. Slides counterstained with Mayer's haematoxylin were then routinely mounted. For immunohistochemistry evaluation, we considered as positive internal control the presence of at least two positive staining plasma cells for each antibody in at least two portal tracts. We counted the number of plasma cells containing IgG and IgM in the three most representative portal tracts for each specimen, calculated the mean values of the number of plasma cells present in the three most representative portal tracts, and evaluated the ratio between mean IgG and IgM plasma cells.

\subsection{Statistical methods}

Continuous variables were summarized as mean $\pm S D$, or median and range when appropriate, and categorical variables as frequency and percentage. Significant differences were calculated using the ANOVA or Kruskal-Wallis test for continuous variables, while a $\chi^{2}$ test was used for categorical variables. Correlation coefficients were calculated by Spearman's test. Multiple linear regression analysis was performed through SPSS software (SPSS Inc., Chicago, IL, USA). 
Table 1

Clinical, biochemical and histological features of patients.

\begin{tabular}{|c|c|c|c|c|c|c|}
\hline Variables & $\operatorname{PBC}(n=40)$ & $\mathrm{AIH}(n=20)$ & $\operatorname{PSC}(n=13)$ & Variant syndromes $(n=10)$ & $\mathrm{CHC}(n=34)$ & $p$ \\
\hline Mean age (years) & $49.53 \pm 11.89$ & $47.53 \pm 19.21$ & $48.85 \pm 21.43$ & $52.10 \pm 14.27$ & $51.45 \pm 11.73$ & 0,9 \\
\hline Female gender & $37(92.5)$ & $15(75)$ & $4(31)$ & $10(100)$ & $15(44)$ & $<0.001$ \\
\hline ALT (IU/ml, UNL) & $2.04 \pm 1.55$ & $8.72 \pm 10.88$ & $1.97 \pm 1.56$ & $3.63 \pm 2.79$ & $2.22 \pm 2.41$ & $<0.001$ \\
\hline GGT (IU/ml, UNL) & $8.01 \pm 12.04$ & $3.92 \pm 4.18$ & $4.30 \pm 5.03$ & $6.45 \pm 4.63$ & $0.71 \pm 0.66$ & 0.003 \\
\hline $\mathrm{AP}(\mathrm{IU} / \mathrm{ml}, \mathrm{UNL})$ & $3.24 \pm 3.81$ & $1.15 \pm 0.90$ & $1.67 \pm 1.05$ & $2.41 \pm 2.61$ & $0.53 \pm 0.41$ & $<0.001$ \\
\hline - Autoantibodies no AMA overall & $14(35)$ & $20(100)$ & $8(66.7)$ & $9(90)$ & $13(38.2)$ & 0.1 \\
\hline - Anti nuclear & $14(35)$ & $18(90)$ & $5(38.5)$ & $6(60)$ & $8(23.5)$ & \\
\hline - Anti smooth muscle & $1(2.5)$ & $8(40)$ & $5(38.5)$ & $7(70)$ & $2(5.9)$ & \\
\hline Anti liver/kidney microsomal & 0 & $3(15)$ & 0 & 0 & $4(11.7)$ & \\
\hline Anti-mitochondrial Ab/M2 & $38(95)$ & 0 & 0 & $3(30)$ & $1(2.9)$ & $<0.001$ \\
\hline Median serum IgM (mg/dl) ${ }^{\mathrm{a}}$ & $395.00(167-3576)$ & $257.00(100-366)$ & $111.0(80-220)$ & $348.00(130-560)$ & $109.0(78-200)$ & 0.001 \\
\hline \multicolumn{7}{|l|}{ Histological features } \\
\hline Interface hepatitis $>1^{\mathrm{b}}$ & $12(30)$ & $13(65)$ & $2(15.4)$ & $7(70)$ & $10(29.4)$ & 0.42 \\
\hline Fibrosis $>1^{\mathrm{b}}$ & $8(20)$ & $5(25)$ & 0 & $2(20)$ & $8(23.5)$ & 0.5 \\
\hline Ductular changes & $37(92.5)$ & $10(50)$ & $9(69.2)$ & $8(80)$ & $7(20.6)$ & $<0.001$ \\
\hline Orcein-positive granules & $21(52.5)$ & 0 & $3(23.1)$ & $4(40)$ & $5(14.7)$ & $<0.001$ \\
\hline Granulomas & $23(57.5)$ & $4(20)$ & 0 & $5(50)$ & 0 & $<0.001$ \\
\hline
\end{tabular}

Continuous variables: mean \pm standard deviation or median (min-max); categorical variables: absolute value (\%).

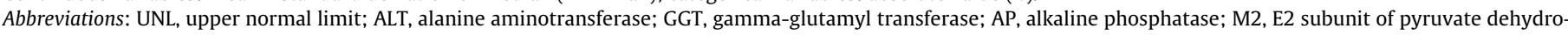
genase mitochondrial auto-antigen.

a Normal range: $40-240 \mathrm{mg} / \mathrm{dl}$.

b According to histological grading and staging system explained in ref. [6].

The ROC curve of the IgM plasma cells counts was performed using MedCalc ${ }^{\circledR}$ software (version 8.0; Mariakerke, Belgium). The box plots were generated through GraphPad Prism ${ }^{\circledR}$ software (version 4.02; San Diego, CA, U.S.A). Differences were considered significant for $p$ values of $<0.05$.

\section{Results}

\subsection{Clinical and histological features of patients}

Demographic, biochemical, serological and histological features of patients at baseline are shown in Table 1 . Forty patients who satisfied the diagnosis of $\mathrm{PBC}, 20$ patients with diagnosis of $\mathrm{AIH}$, 13 patients with a diagnosis of PSC and 10 patients with VS were compared to 34 patients with a diagnosis of $\mathrm{CHC}$, according to the criteria mentioned above. Female gender was prevalent in PBC and in VS compared to other groups $(p<0.001)$. ALT levels were significantly higher in patients with $\mathrm{AIH}$ compared to those of other groups (AIH: 8.72 UNL; PBC: 2.04 UNL; PSC: 1.97 UNL; VS: 3.63 UNL and CHC: $2.22 \mathrm{UNL} \mathrm{IU/ml;} p<0.001)$. Conversely, GGT levels were higher in $\mathrm{PBC}$ and in VS patients compared to the other two groups (PBC: 8.01 UNL; VS: 6.45 UNL; AIH: 3.92 UNL; PSC: 4.30 UNL and CHC: $0.71 \mathrm{UNL} \mathrm{IU} / \mathrm{ml} ; p<0.003)$. Likewise, AP levels were significantly more elevated in PBC and in VS patients (PBC: 3.24 UNL; VS:
2.41 UNL; AIH: 1.15 UNL; PSC: 1.67 UNL and CHC: 0.53 UNL IU/ml; $p<0.001$ ).

Autoantibodies were present in all patients with $\mathrm{AIH}$, in $90 \%$ of patients with VS and in 67\% of those with PSC. AMA-M2 was found in patients with $\mathrm{PBC}(95 \%)$, in VS patients $(30 \%)$ and in only one patient with $\mathrm{CHC}(3 \%)(p<0.001)$. We found the positivity of antinuclear antibody (ANA) with indirect immunofluorescence method in 14 of our PBC patients: a centromer pattern in 7 patients, a nucleolar pattern in 3 patients, a membranous in 3 and a multi-nuclear dots pattern in one patient.

More elevated median serum IgM levels were found in PBC and in VS patients ( $395 \mathrm{mg} / \mathrm{dl}$ and $348 \mathrm{mg} / \mathrm{dl}$, respectively) compared to those observed in AIH ( $257 \mathrm{mg} / \mathrm{dl})$, while in PSC and CHC patients we observed normal levels of serum IgM $(p=0.001)$.

A moderate-severe grade of interface hepatitis in liver biopsy was more frequently observed in VS and in $\mathrm{AIH}$ patients (70\% and $65 \%$, respectively) compared to PBC (30\%), to CHC (29\%) and PSC patients (15\%), though with no significant differences $(p=0.42)$. We found a similar rate of moderate-severe stage of fibrosis in PBC, $\mathrm{AIH}, \mathrm{VS}$ and $\mathrm{CHC}$ patients (from 20 to $25 \%$ of the patients), but in none of the PSC patients $(p=0.5)$. Ductular changes were prevalent in $\mathrm{PBC}$ and in VS patients (92.5\% and $80 \%$, respectively) compared to $\mathrm{AIH}(50 \%)$ and PSC (69\%); we found this feature only in $21 \%$ of CHC patients $(p<0.001)$. Orcein-positive granules were observed
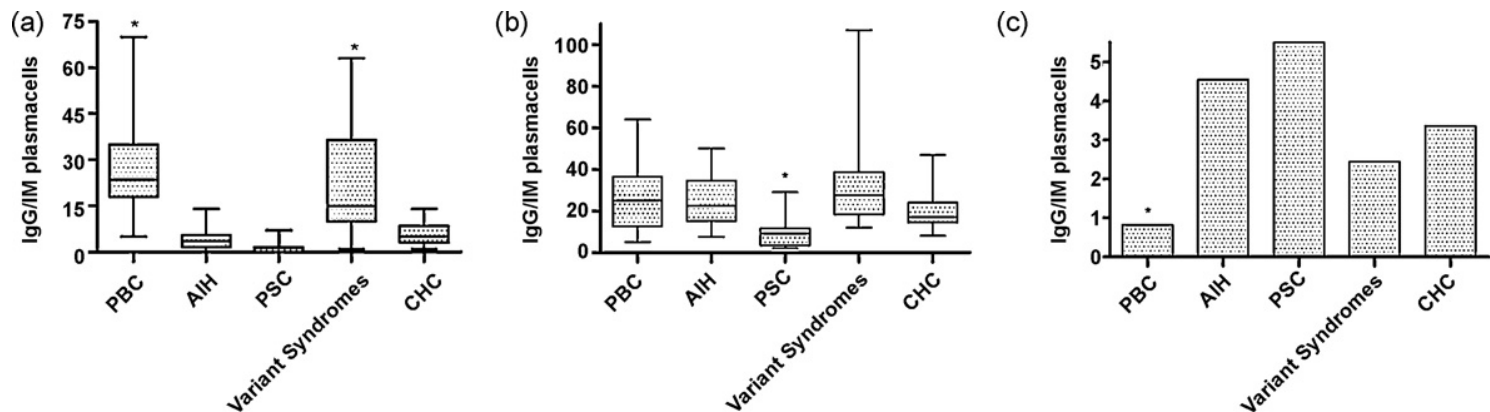

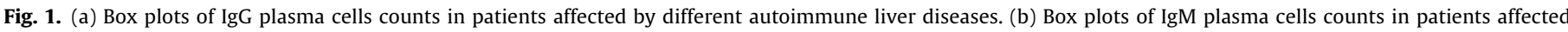

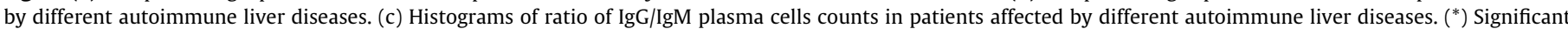
difference in the comparison with the other groups. 


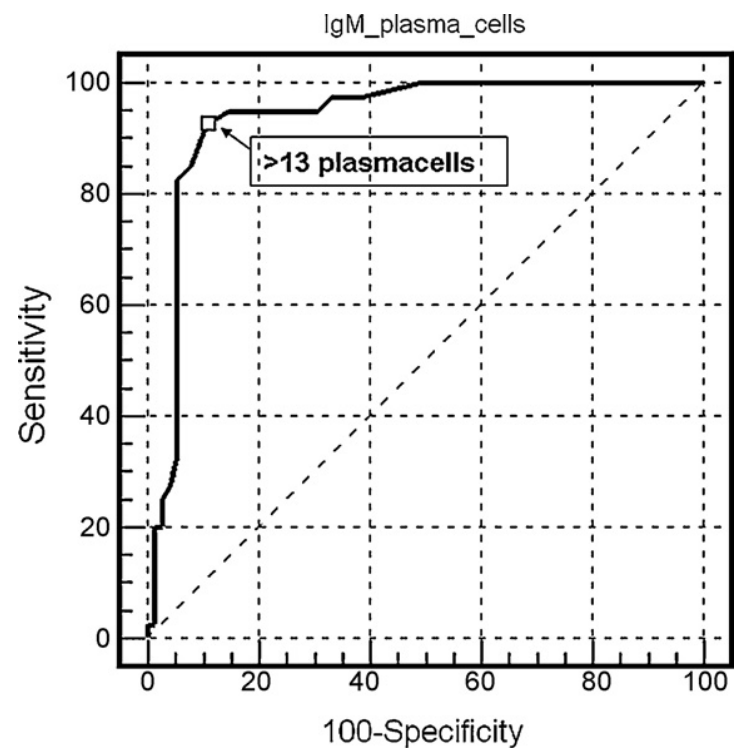

Fig. 2. ROC curve of IgM plasma cells counts according to clinical/histological diagnosis of PBC. Cut-off point: >13 mean IgM plasma cells in the portal tract of liver biopsy (sensitivity $92.5 \%$; specificity $89.3 \%$ ). Area under the ROC curve: 0.94 (95\% confidence interval $0.87-0.97 ; p<0.001$ ).

in $52.5 \%$ of the patients with PBC, in $40 \%$ of those with VS, but in only $23 \%$ of the patients with PSC and in $15 \%$ of those with CHC, $(p<0.001)$. No patients with AIH showed this feature in liver biopsy. Likewise, epithelioid granulomas were found more frequently in $\mathrm{PBC}$ and in VS patients as compared to others (PBC: $57.5 \%$; VS: $50 \%$; AIH: $20 \%$; PSC and CHC: $0 \%)(p<0.001)$.

\subsection{Analysis of IgM and IgG plasma cells counts in different autoimmune liver diseases}

The median value of IgG plasma cells mean-counts observed in the portal spaces of liver biopsies was significantly lower in the PSC group (9 cells/portal tract) as compared to the PBC group ( 25 cells/portal tract), to AIH (24.5 cells/portal tract), to VS (27.5 cells/portal tract) and to CHC patients (17 cells/portal tract) respectively $(p<0.001)$ (Fig. 1a). The median value of IgM

Table 2

Spearman's rho correlation analysis of IgM and IgG plasma cells scores and clinical, serological and histological features of patients.

\begin{tabular}{|c|c|c|c|c|}
\hline \multirow[t]{2}{*}{ Variables } & \multicolumn{2}{|l|}{ IgM plasma cells } & \multicolumn{2}{|l|}{ IgG plasma cells } \\
\hline & Spearman's rho & $p$ & Spearman's rho & $p$ \\
\hline Age & -0.04 & 0.64 & 0.04 & 0.68 \\
\hline Gender & 0.38 & $<0.001$ & 0.14 & 0.16 \\
\hline ALT UNL & -0.05 & 0.56 & 0.25 & 0.01 \\
\hline GGT UNL & 0.35 & 0.001 & 0.19 & 0.05 \\
\hline AP UNL & 0.36 & 0.001 & 0.11 & 0.26 \\
\hline Autoantibodies & -0.11 & 0.27 & 0.22 & 0.03 \\
\hline AMA-M2 & 0.66 & $<0.001$ & 0.14 & 0.24 \\
\hline Serum $\operatorname{IgM}^{\mathrm{a}}$ & 0.58 & $<0.001$ & 0.22 & 0.19 \\
\hline Interface hepatitis $>1^{b}$ & 0.03 & 0.76 & 0.28 & 0.005 \\
\hline Fibrosis $>1^{\mathrm{b}}$ & 0.08 & 0.43 & 0.25 & 0.02 \\
\hline Ductular changes & 0.45 & $<0.001$ & 0.18 & 0.09 \\
\hline Orcein granules & 0.51 & $<0.001$ & 0.23 & 0.04 \\
\hline Granulomas & 0.45 & $<0.001$ & 0.22 & 0.06 \\
\hline
\end{tabular}

Abbreviations: UNL, upper normal limit; ALT, alanine aminotransferase; GGT, gamma-glutamyl transferase; AP, alkaline phosphatase; AMA, anti-mitochondrial antibody; M2, E2 subunit of pyruvate dehydrogenase mitochondrial auto-antigen.

a Normal range: $40-240 \mathrm{mg} / \mathrm{dl}$.

b According to histological grading and staging system explained in ref. [6].

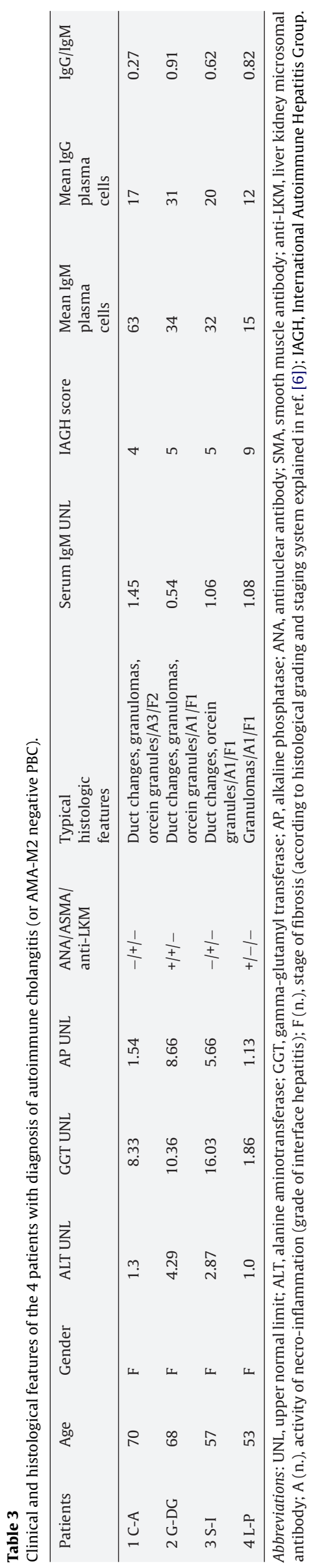




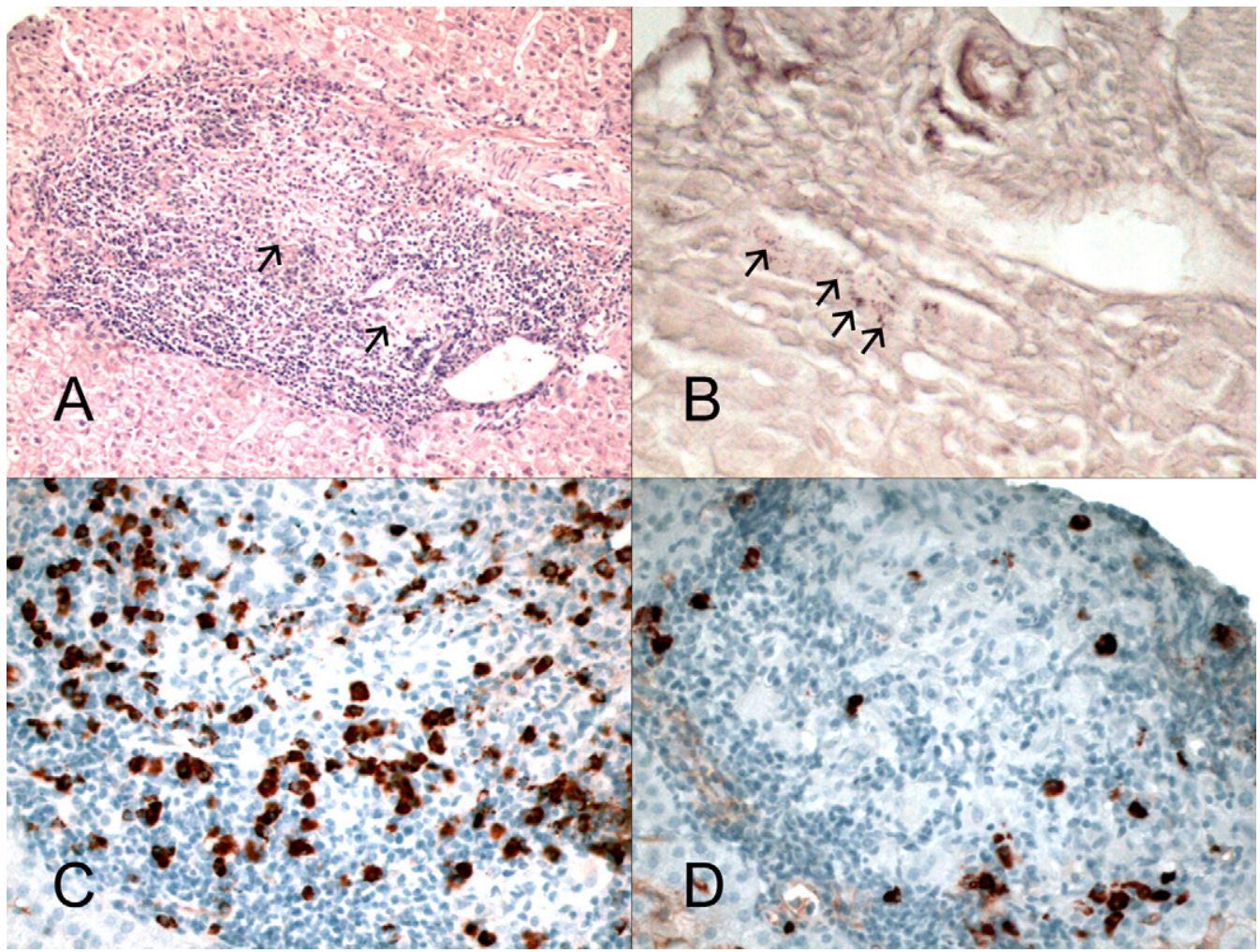

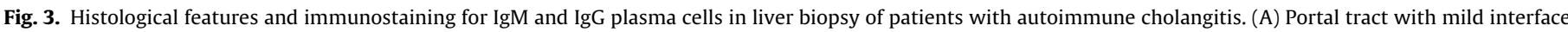

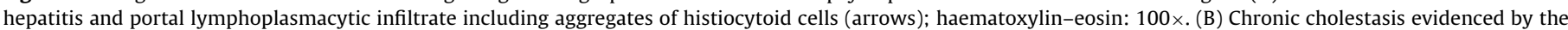

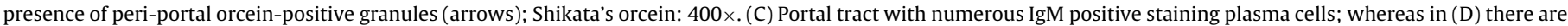
a few IgG positive staining plasma cells; immunostaining $250 \times$.

plasma cells mean-counts was significantly higher in PBC (about 23.5 cells/portal tract) and in VS group (15 cells/portal tract), as compared to those found in AIH (4 cells/portal tract), in PSC (2 cells/portal tract) and in CHC group ( 5 cells/portal tract) $(p<0.001)$, with no significant differences between PBC and VS patients $(p=0.2)$ (Fig. 1b). The ratio of the median values of IgG/IgM plasma cells mean-counts was below 1 only in the PBC group (Fig. 1c) $(p<0.001)$.

The predictive strength of the IgM plasma cells mean-count for the diagnosis of PBC was evaluated by ROC curve, showing that the best cut-off to discriminate the diagnosis of PBC was above 13 IgM plasma cells, with an area under the curve of 0.94 (95\% C.I.: $0.87-0.97 ; p<0.001$ ) (Fig. 2). The sensitivity at this cut-off was 92.5\% (95\% C.I.: 79.6-98.3) and the specificity 89.3\% (95\% C.I.: 80.1-95.3), with positive and negative predictive values of $82.2 \%$ (95\% C.I.: 67.4-91.5) and 95.7\% (95\% C.I.: 87.2-98.9), respectively.

The correlations between IgM and IgG plasma cells mean-counts in the portal infiltrate of the liver tissue with biochemical, serological and histological variables is shown in Table 2. By Spearman's rho coefficient of correlation, the mean count of IgM plasma cells in portal tracts was significantly correlated with female gender (rho $0.38 ; p<0.001$ ), serum GGT values (rho 0.35; $p=0.001$ ), AP levels (rho 0.36; $p=0.001$ ), presence of AMA-M2 (rho 0.66; $p<0.001$ ) and serum IgM levels (rho $0.58 ; p<0.001$ ). Moreover the presence of IgM plasma cells in portal tracts correlated with bile duct changes, (rho $0.45 ; p<0.001$ ), orcein-positive granules (rho $0.51 ; p<0.001$ ) and granulomas (rho $0.45 ; p<0.001$ ) on liver biopsy. By multiple linear regression analysis the natural logarithm of IgM plasma cells mean-counts resulted independently associated only with serum GGT values $(p<0.001)$.

Conversely, IgG plasma cells mean-counts were correlated with high levels of ALT (rho $0.25 ; p=0.01$ ), with GGT values (rho
$0.19 ; p=0.05$ ), presence of autoantibodies (rho $0.22 ; p=0.03$ ) and with the following features on liver biopsy: presence of a moderate-severe interface hepatitis, (rho 0.28; $p=0.005$ ), and fibrosis (rho $0.25 ; p=0.02$ ), and finally with presence of orcein granules (rho $0.23 ; p=0.04$ ) but only with ALT levels by multivariate analysis $(p=0.02)$.

\section{IgM and IgG plasma cells count in liver biopsy of patients with variant syndromes}

\subsection{Patients with autoimmune cholangitis}

Clinical and histological features, including IgM and IgG plasma cells mean-counts of the inflammatory infiltrate in liver biopsies of 4 patients with a diagnosis of AIC are reported in Table 3. These patients had elevated values of GGT and AP, met at least 2 criteria for diagnosis of $\mathrm{PBC}$, but had negative values for AMA-M2, and had a IAHG score for diagnosis of $\mathrm{AIH}<10$. We found a count of IgM plasma cells higher than 13 in all patients (Fig. 3 ). It is worth noting that IgG plasma cells were prevalent in patients with a more marked cytolytic activity, though the ratio of IgG/IgM plasma cells meancounts was below 1 for all these patients.

\subsection{Patients with overlap syndrome}

Clinical and histological features of the 6 patients with overlap syndrome are summarized in Table 4 . These patients met at least two of the three criteria for diagnosis of PBC, and had an IAHG score of at least 10 points, compatible with a probable/definitive diagnosis of AIH. Two of them resulted negative for AMA-M2, but showed histological features suggestive of $\mathrm{PBC}$, such as presence of florid bile duct lesions with duct proliferation, and a lymphoplasmacytic 


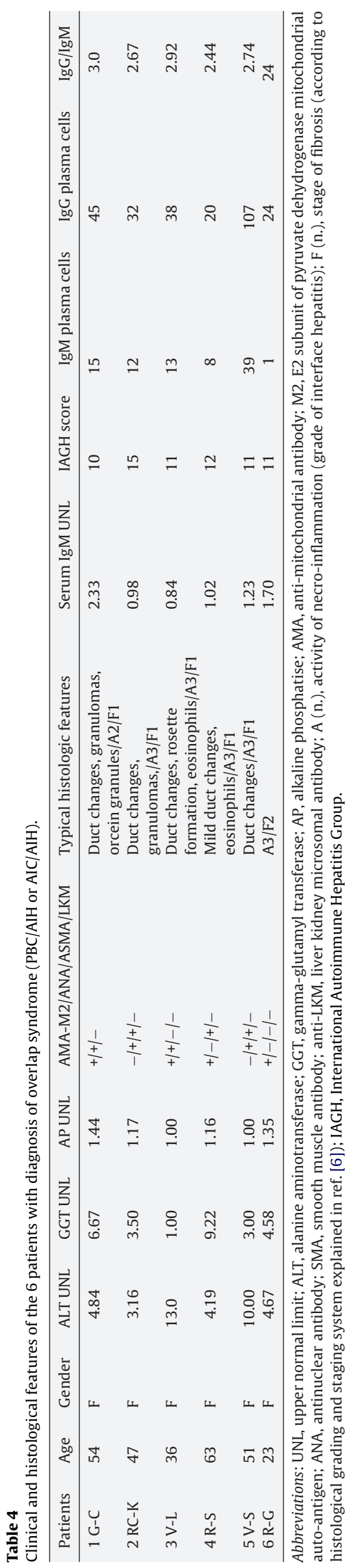

infiltrate associated with severe peri-portal and lobular necroinflammation. Moreover they simultaneously showed a chronic elevation of ALT and GGT, and the presence of ANA and ASMA, reaching an aggregate IAHG score compatible with a definitive diagnosis of AIH (revised IAHG score of 15) and a probable diagnosis of AIH (revised IAHG score of 11). This justified the diagnosis of OS (autoimmune cholangitis/AIH) for these two patients. The other 4 patients had a typical pattern of PBC/AIH overlap, simultaneously showing features of both diseases.

Regarding the type of plasma cells observed in the portal spaces of the liver biopsy, a ratio of IgG/IgM plasma cells mean-counts above 1 was found in all patients (Fig. 4).

\section{Discussion}

This study confirms the relevance of characterization of the immunoglobulin isotype of plasma cells infiltrating the liver parenchyma of patients with autoimmune liver diseases. We found, specifically in liver biopsy of patients with a definite diagnosis of PBC, a marked amount of IgM plasma cells in the portal and peri-portal tracts compared to patients with $\mathrm{AIH}$ and PSC such as to patients with $\mathrm{CHC}$, so showed that this finding significantly correlated with every typical clinical and histological feature of PBC. Conversely, IgG plasma cells were observed mostly in cases characterized by a more pronounced inflammatory activity, for example those with a diagnosis of $\mathrm{AIH}$.

These findings are in line with the evidence documented nearly 20 years ago by Milne and Horne [8], and with that of a recent study by Daniels et al. [9], which demonstrates that immunostaining for IgM and IgG plasma cells in liver biopsy can be helpful in differentiating PBC from AIH. In our study of a larger cohort of patients with autoimmune liver diseases, we aimed to explore the usefulness of this finding in the more difficult-to-define cases of VS, where a remarkable difference was found in the relative expression of IgM and IgG plasma cells (IgG/IgM ratio) between cases of AIC and those with OS of PBC/AIH.

When suspecting an autoimmune liver disease, biopsy is an important tool not only for the staging but also for differential diagnosis, particularly in cases in which clinical and laboratory features are ambiguous. However, some histological findings, such as plasma cells infiltration and piecemeal necrosis are seen in several diseases, for example in AIH and chronic hepatitis C (CHC) [14]. Similarly, lesions considered as representative of $\mathrm{PBC}$, such as bile duct changes and epithelioid granulomas, have modest disease specificity. In fact, bile duct injuries are also reported in about $30 \%$ of AIH patients [18] and frequently in CHC [14], and granulomas are found in other conditions, such as sarcoidosis, drug-induced liver injury and, occasionally, in CHC $[19,20]$. In our study we observed ductular changes in about $92 \%$ of $\mathrm{PBC}$ patients, but in $50 \%$ of $\mathrm{AIH}$ in $69 \%$ of PSC and also in $21 \%$ of CHC patients, as well. In addition, granulomas, observed in about $57 \%$ of PBC patients, were also found in $20 \%$ of $\mathrm{AIH}$ patients. Orcein-positive granules were found in only $52 \%$ of $\mathrm{PBC}$ patients, in $23 \%$ of patients with PSC and in $15 \%$ of those with $\mathrm{CHC}$.

In a small proportion of patients (5-10\%) who show features on the whole suggestive of $\mathrm{PBC}$, but without detectable serum AMA and/or M2 (AIC), the diagnosis can be difficult to make, particularly when the histological features are ambiguous. Moreover, in the cases characterized by mixed clinical and histological features of two diseases, such as OS, a histological misunderstanding may be generated, with relevant consequences for therapeutic decisions. Thus, an additional histological element able to discriminate among these different autoimmune liver diseases, principally in the doubtful cases, is desirable. 


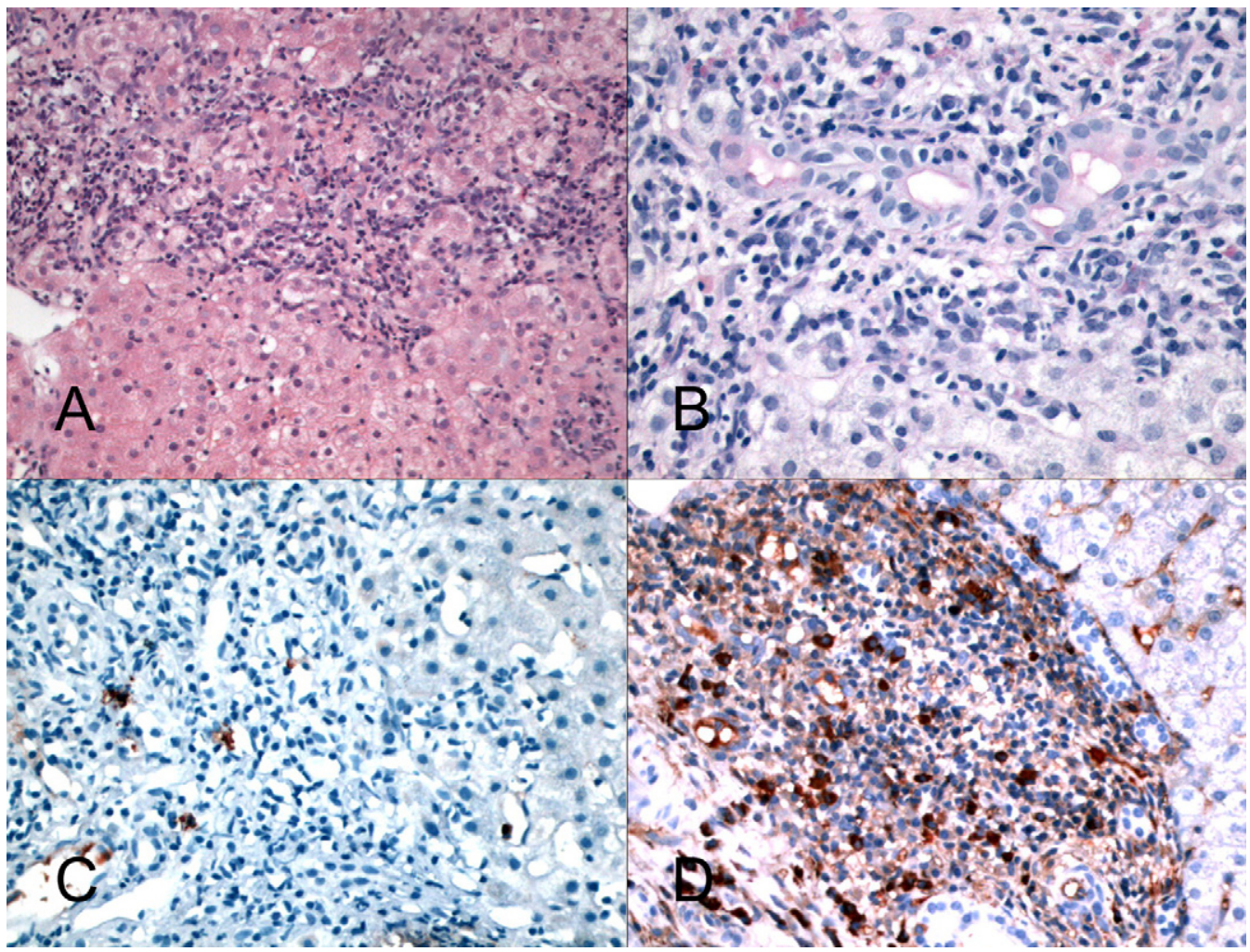

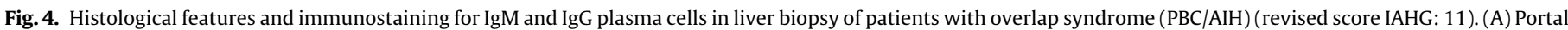

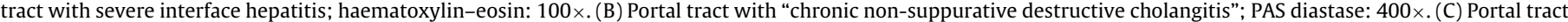
with a few IgM positive staining plasma cells while in (D) IgG positive staining plasma cells are numerous; immunostaining $250 \times$.

In this study a more conspicuous IgM plasma cells infiltrate was specific for PBC, and a ratio of IgG/IgM plasma cells mean-counts of less than 1 was found only in this group of patients and in the subgroup of patients with a diagnosis of AIC. This pathological entity, which the older literature considered distinct from $\mathrm{PBC}$, is now firmly accepted as a variant of PBC [5]. This is in line with our observation of the analogous behaviour of plasma cells immunoglobulin expression. Therefore, independently of the presence of autoantibodies and ALT levels, we prefer to treat these patients solely with UDCA, as is done with AMA-M2 positive PBC patients.

In the subgroup of patients with a diagnosis of overlap $\mathrm{PBC} / \mathrm{AIH}$ or $\mathrm{AIC} / \mathrm{AIH}$, we found a value of the IgG/IgM ratio higher than 1 in all of them because they showed a more pronounced inflammatory activity, explained by biochemical and histological features, evidently related to the predominance of IgG plasma cells on liver biopsy. For these patients we prefer a combined therapeutic approach with steroids and UDCA.

Elevated serum IgM levels have been well described as a typical clinical finding of PBC [11], and in our cohort, patients with PBC showed higher serum IgM values with respect to the others. However, this parameter in our cohort was unable to discriminate $\mathrm{PBC}$ from $\mathrm{AIH}$ and/or the different subgroups of VS.

Our study thus provides further evidence of the efficacy of this additional histological evaluation, specifically for ambiguous cases of autoimmune liver diseases, such as the variant and mixed syndromes.

\section{Contributors}

D. Cabibi designed the study and contributed to data acquisition. G. Tarantino was responsible for writing the manuscript and for statistical analyses. V. Di Marco and A. Craxì (Director of the GI \& Liver Unit) were responsible for writing the manuscript, and have seen and approved the final version. F. Barbaria and M. Campione performed laboratory procedures. All authors have seen and approved the final version of the manuscript.

\section{Conflict of interest statement}

None declared.

\section{List of abbreviations}

AIC, autoimmune cholangitis; AIH, autoimmune hepatitis; AILD, autoimmune liver disease; AMA, anti-mitochondrial antibody; ANA, antinuclear antibody; AP, alkaline phosphatase; GGT, gamma-glutamyl transferase; LKM, liver kidney microsomal type 1 antibody; M2, E2 subunit of pyruvate dehydrogenase mitochondrial auto-antigen; OS, overlap syndrome; PBC, primary biliary cirrhosis; PSC, primary sclerosing cholangitis; SMA, smooth muscle antibody; VS, variant syndrome.

\section{Acknowledgement}

The authors would like to thank Warren Blumberg for his help in editing this paper.

\section{References}

[1] Batts KP, Ludwig J. Histopathology of autoimmune hepatitis, primary biliary cirrhosis and primary sclerosing cholangitis. In: Krawitt EL, Wiesner RH, Nishioka M, editors. Autoimmune liver diseases. 2nd ed. Amsterdam: Elsevier; 1998. p. $115-40$.

[2] Rust C, Beuers U. Overlap syndromes among autoimmune liver diseases. World J Gastroenterol 2008;14:3368-73. 
[3] Beuers U. Hepatic overlap syndromes. J Hepatol 2005;42:S93-9.

[4] Beuers U, Rust C. Overlap syndromes. Semin Liver Dis 2005;25:311-20.

[5] Hirshfield GM, Heathcote EJ. Antimitochondrial antibody-negative primary biliary cirrhosis. Clin Liver Dis 2008;12:323-31.

[6] Hiramatsu K, Aoyama H, Zen Y, et al. Proposal of a new staging and grading system of the liver for primary biliary cirrhosis. Histopathology 2006;49:46678.

[7] Portmann BC, Nakanuma Y. Diseases of the bile ducts. In: MacSween RNM, Burt AD, Portmann BC, Ishak KG, Scheuer PJ, Anthody PP, editors. Pathology of the liver. 4th ed. London: Churchill Livingstone; 2001. p. 435-506.

[8] Milne DS, Horne $\mathrm{CH}$. Immunoglobulin classes in biopsies of autoimmune liver disease. Histopathology 1990;16:283-6.

[9] Daniels JA, Torbenson M, Anders RA, et al. Immunostaining of plasma cells in primary biliary cirrhosis. Am J Clin Pathol 2009;131:243-9.

[10] Romero-Gómez M, Wichmann I, Crespo J, et al. Serum immunological profile in patients with chronic autoimmune cholestasis. Am J Gastroenterol 2004;99:2150-7.

[11] Kikuchi K, Lian Z-X, Yang G-X, et al. Bacterial CpG induces hyper-IgM production in CD27 memory B cells in primary biliary cirrhosis. Gastroenterology 2004;128:304-12.

[12] Chapman RW. Role of immune factors in the pathogenesis of primary sclerosing cholangitis. Semin Liver Dis 1991;11:1-4.
[13] Sherlock S, Dooley J, editors. Diseases of the liver and biliary system. 10th ed Oxford: Blackwell Science Ltd; 1997. p. 239-52.

[14] Scheuer PJ, Lefkowitch JH, editors. Liver biopsy interpretation. 6th ed. London: W.B. Saunders Co. Ltd; 2000. p. 166-7.

[15] Alvarez F, Berg PA, Bianchi FB, et al. International Autoimmune Hepatitis Group Report: review of criteria for diagnosis of autoimmune hepatitis. J Hepatol 1999;31:929-38.

[16] LaRusso NF, Shneider BL, Black D, et al. Primary sclerosing cholangitis: summary of a workshop. Hepatology 2006;44:746-64.

[17] Talwalkar JA, Keach JC, Angulo P, et al. Overlap of autoimmune hepatitis and primary biliary cirrhosis: an evaluation of a modified scoring system. Am J Gastroenterol 2002;97:1191-7.

[18] Sato Y, Harada K, Sudo Y, et al. Autoimmune hepatitis associated with bile duct injury resembling chronic non-suppurative destructive cholangitis. Pathol Int 2002;52:478-82.

[19] Wiesener RH, La Russo NF, Ludwig J, et al. Comparison of the clinicopathological features of primary sclerosing cholangitis and primary biliary cirrhosis. Gastroenterology 1985;88:108-14.

[20] Nakanuma Y, Ohta G. Quantitation of hepatic granuloma and epithelioid cells in primary biliary cirrhosis. Hepatology 1983;3:423-7. 